\title{
Strategic Spatial Planning Consideration in the Kingdom Vision 2030 and National Physical Planning Plan: Utilizing Content Analysis Methodology for Evaluation and Comparison
}

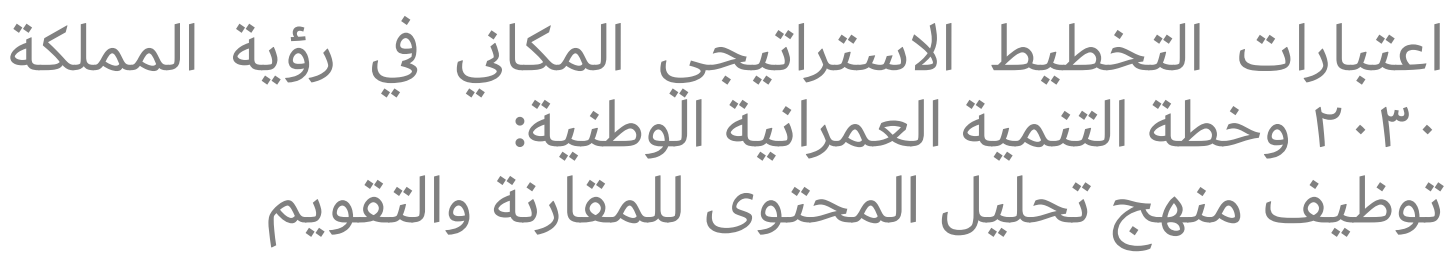

Hussein Ali Alribah and Ahmed Jarallah Al-Jarallah

حسين علي الربح و أحمد جارالله الجار الله

Corresponding Author:

Hussein Ali Alribah

halribh@gmail.com

Received: 28 December 2017

Accepted: 2 February 2018

Published: 1 May 2018

Publishing services provided by Knowledge $\mathrm{E}$

(c) Hussein Ali Alribah and Ahmed Jarallah Al-Jarallah. This article is distributed under the terms of the Creative Commons

Attribution License, which permits unrestricted use and redistribution provided that the original author and source are credited.

Selection and Peer-review under the responsibility of the Urban Planning Iraq Conference Committee.

\section{G OPEN ACCESS}

Department of Urban and Regional Planning, Faculty of Architecture and Planning, Imam Abdulrahman Ibn Faisal University

$$
\text { قسم التخطيط الحضري والإقليمي، كلية العمارة والتخطيط بجامعة الامام عبدالرحمن ابن فيصل }
$$

\section{Abstract}

Urban and regional planning over the time pass through a lot of changes in the 2oth century and have been impacted by economic, social and environmental problems. However, old traditional planning approaches were replaced by sustainable approaches to avoid the limitations and improve the results. One of these new approaches known as strategic spatial planning which defined as a long term perspective with clear methodology and strategic vison for critical and important issues in all aspects of economic, social and environment as well as involve stakeholders in this vision through focusing on spatial aspects to achieve sustainable development.

The aim of this study is to analyze the content of vision 2030 for Saudi Arabia based on the perspective of spatial strategic planning and compare it with the content of urban national strategy through using same base line. The purpose of this comparison is to explore the achievement of spatial development considerations in the 2030 vision. The literatures review helps to identify the key aspects of strategic spatial planning (economic, social, environmental), as well as identify important considerations associated with each one of them. However, eight considerations were associated with the economical aspect and seven considerations were associated with the social aspect. While, there are five considerations associated with the environmental aspect. By applying content analyses approach; the quantitative analysis of repetitions of the spatial considerations was high in the urban strategy rather than vision 2030 in the overview context. Moreover, there is homogeneity for spatial considerations in the urban strategy with more clarity than vision 2030. Based on the repetitions of spatial 
considerations in 2030 vision; the vision is focusing more on the economical aspect, social aspect then environmental aspect.

مر التخطيط الحضري والاقليمي بالعديد من التطورات عبر القرن العشرين، كما أثرت المشاكل الاقتصادية

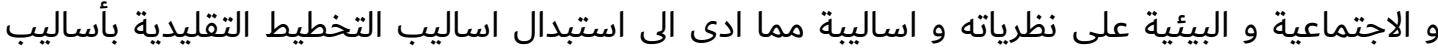

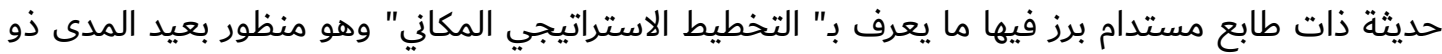

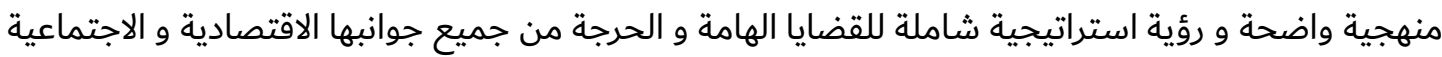
و البيئية إضافةٍ الى إشراك أصحاب المصلحة في تلك الرؤية بالتركيز على الجوانب المكانية مما يساعد في

التنمية المستدامة.

تهدف هذه الدراسة إلى تحليل محتوى رؤية ·ـ ·ـ للمملكة بالمقارنة مع محتوى الاستراتيجية العمرانية من

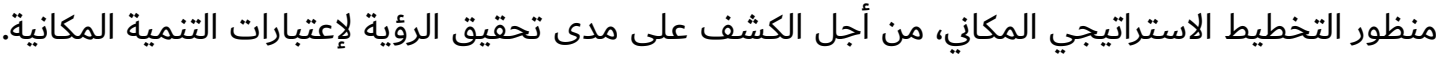
وبمراجعة العديد من الدراسات السابقة امكن التعرف الى الجوانب الرئيسية (الاقتصادية، الاجتماعية، البيئية) التي يقوم عليها التخطيط الاستراتيجي المكاني و كذلك تحديد الاعتبارات الهامة المرتبطة بكل جانب. بالتالي

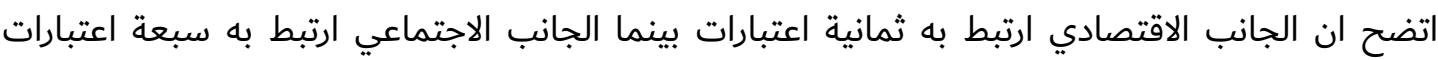
في حين ان الجانب البيئي ارتبط به خمسة اعتبارات. وبتطبيق اسلوب تحليل المحتوى تم تحديد تكرارات

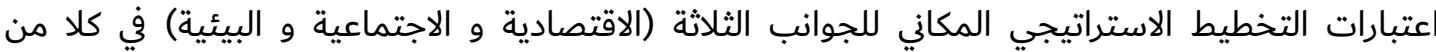

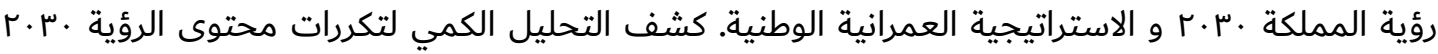
والاستراتيجية العمرانية، بإن الاستراتيجية العمرانية تتفوق بشكل ملحوظ على الرؤية من حيث الاجمالي العام

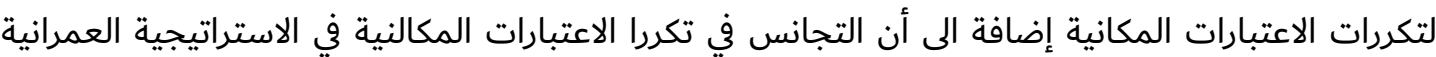

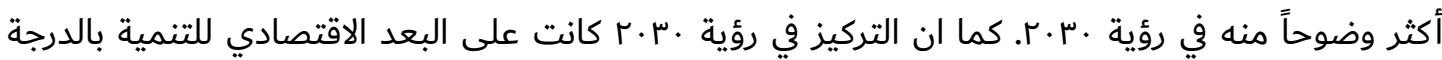

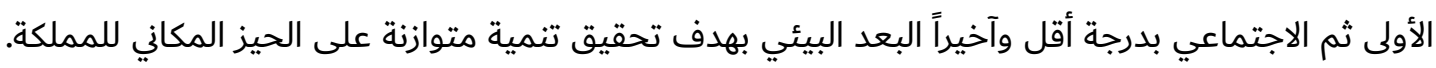

Keywords: Spatial Strategic Planning, 2030 Vision, Kingdom of Saudi Arabia, spatial strategy, Content Analysis, statistical description, Explanatory statistics

التخطيط الاستراتيجي المكاني، رؤية ·ـ·rr، المملكة العربية السعودية، الاستراتيجية

المكانية، تحليل المحتوى، الوصف الاحصائي، الاحصاء الاستدلالي.

أن الإهتمام الكبير في التخطيط الإستراتيجي في المملكة العربية السعودية برز في العديد من الخطط الإستراتيجية في الآونة الأخيرة، حيث صدرت عدد من الاستراتيجيات المكانية والقطاعية منذ بداية الالفية

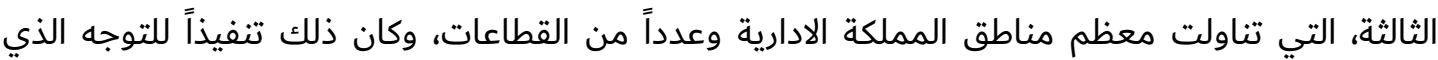
ظهر بوضوح في خطط التنمية الخمسية مؤخراً وهو ما أشارت إليه خطة التنمية التاسعة من الإن من التتقال في 
الفكر التنموي قصير ومتوسط المدى إلى التخطيط الاستراتيجي طويل المدى، وزيادة الاهتمام بالبعد المكاني للتنمية من خلال التركيز على التنمية المتوازنة بين المناطق. وبذلك فإن التخطيط الاستراتيجي المكاني

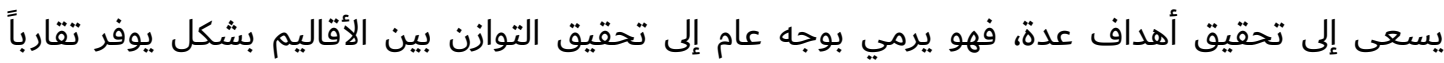

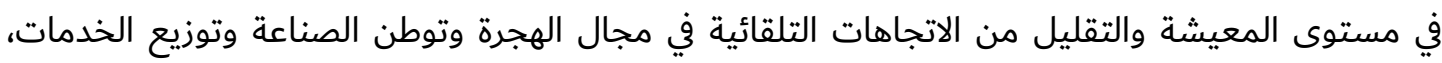

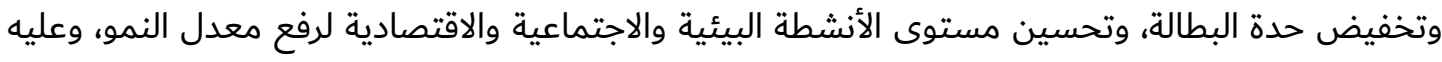

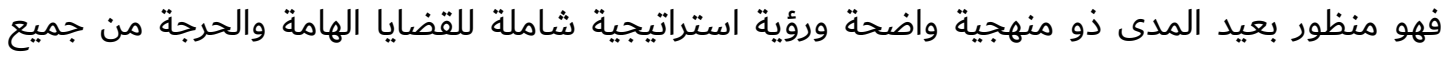

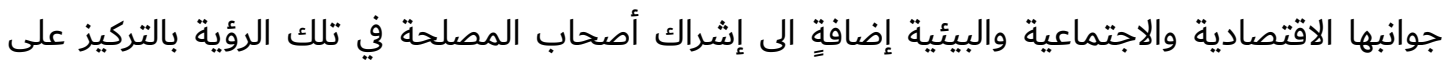

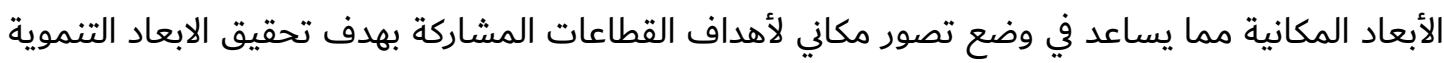
الاقتصادية والاجتماعية و البيئية و الوصول الى التنمية مكانية المستدامة.

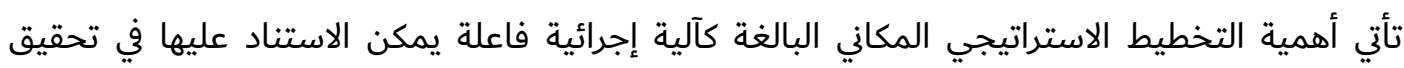

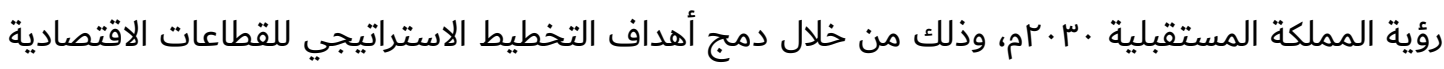

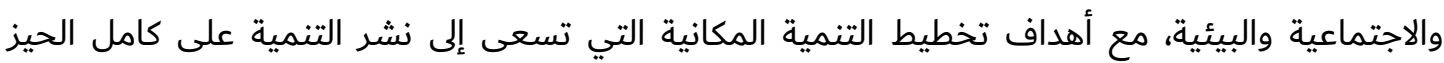

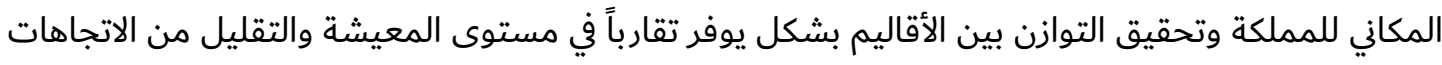
التلقائية في مجال الهجرة وتوطن الصناعة وتوزيع الخدمات، وتخفيض لحفئ حدة البطالة، وتحسين مستوى الأنشطة

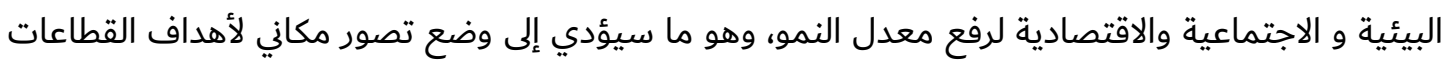

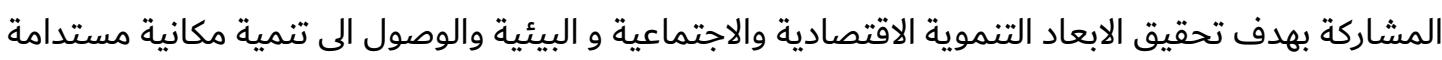

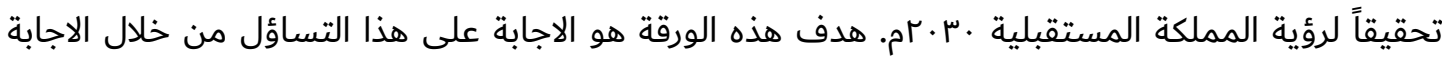

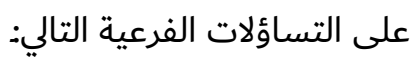

ا. ما اعتبارات التخطيط الاستراتيجي المكاني المهمة التي يُمكن أستشفافها من أدبيات التخطيط

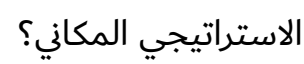

r. ما قيم مقاييس الوصف الإحصائي لتكرارات اعتبارات التخطيط الاستراتيجي المكاني في محتوى رؤية

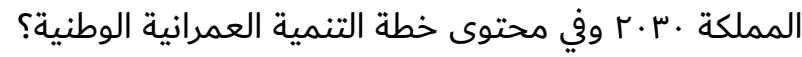

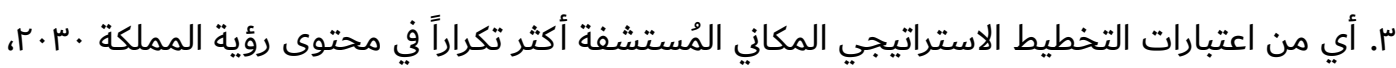
وفي محتوى خطة التنمية العمرانية الوطنية ؟ ع. هل هناك فروق ذات دلالة إحصائية بين تكرارات اعتبارات التخطيط الاستراتيجي المكاني بين خطة

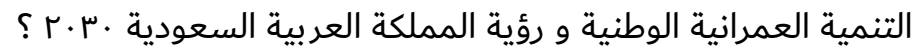

بناء على تساؤلات الدراسة وخلاصة الدراسات السابقة، فإن هدف البحث النهائي سيحقق من خلال تحقيق

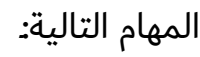

ا. استشفاف اعتبارات التخطيط الاستراتيجي المكاني من خلال مراجعة الاطر والمفاهيم النظرية للتخطيط

$$
\text { الاستراتيجي والتنمية المكانية. }
$$

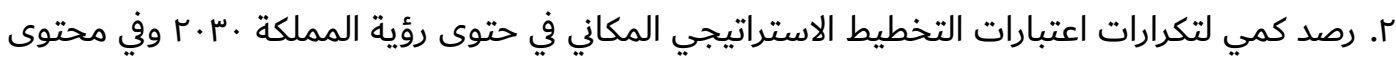
خطة التنمية العمرانية الوطنية للمملكة العربية السعودية. 


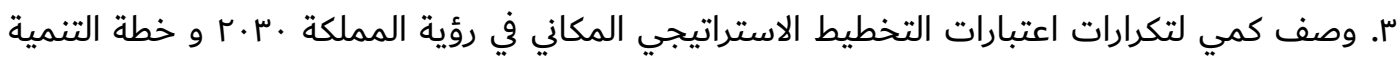
العمرانية الوطنية للمملكة العربية السعودية.

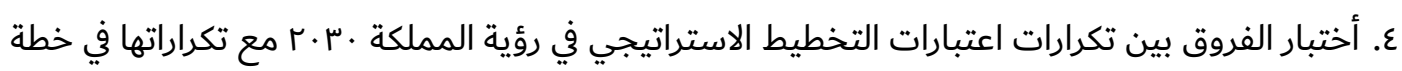
التنمية العمرانية الوطنية للمملكة العربية السعودية وتحديد دلالتها الاحصائية.

للإجابة على السؤال الاول وتحقيق الهدف الاول للبحث المتمثل في تكوين إطار نظري للدراسة ستركز

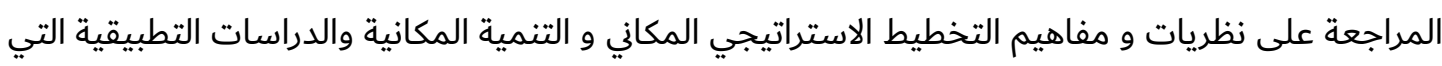

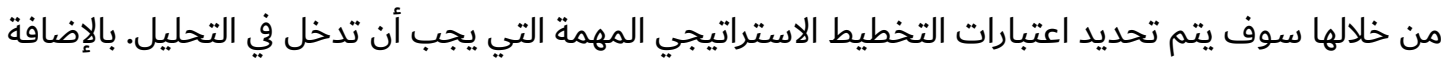

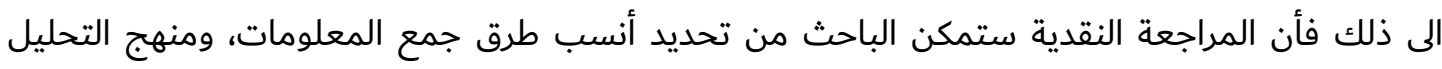
المناسب الذي يحقق هدف البحث وتمكنه من إنجازه.

\section{1-1. إ مفهوم التنمية المكانية وهدافها}

التنمية المكانية Spatial Development تعني"بلوغ الهيكل المكاني للتنمية في أي مكان وفي أي زمان

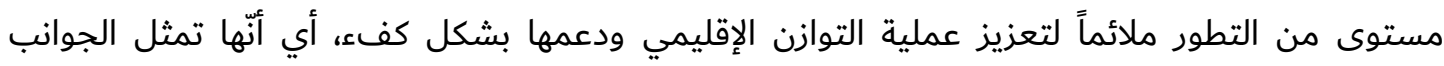

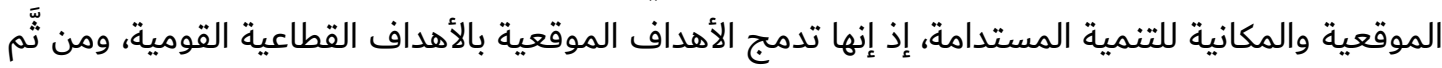

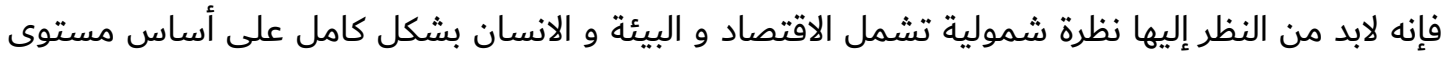

الحيز المكاني (Hansen 1968). والتنمية المكانية تسعى الى تحقيق اهداف عدة، حيث أنها ترمي بوجه المانه عام إلى تحقيق التوازن بين الأقاليم

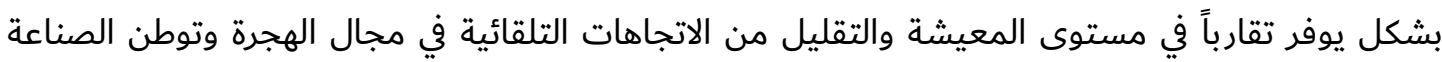

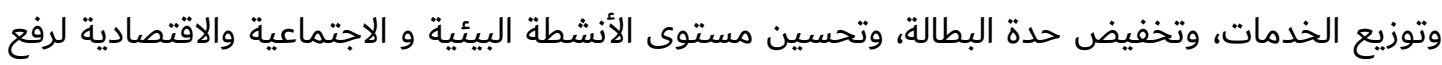
معدل النمو، ويُمكن حصر تلك الأهداف في النقاط التالية: أ- -تحقيق التوازن الأقليمي بحيث لا يكون هناك تأثيرات سلبية على نمو وتطور الأقاليم أو المناطق المجاورة كإستنزاف الإمكانات التنموية أو هدر الموارد. ب- -الإرتقاء بمستويات التنمية في الاقاليم، وذلك من خلال رفع معدلات النمو في الجوانب الهامة المرتبطة للإرتقاء بمستويات المعيشة، وتحقيق الرفاهية الاقتصادية والاجتماعية مما يؤدي الى التنمية المستدامة. ج- -رفع مستوى الإستخدام النوعي والكمي في الاقاليم والتقليل من حركة الهجرة الداخلية للسكان ما بين الأقاليم، ولا سِيمَّا الهجرة من الريف إلى المدينة، وبذلك تقلل ظاهرة ترييف المدينة من الانتشار. د- -توليد تجمعات حضرية، فالتحضر يعني "زيادة سكان المدن عن طرق الانتقال من الريف إلى المدن أو

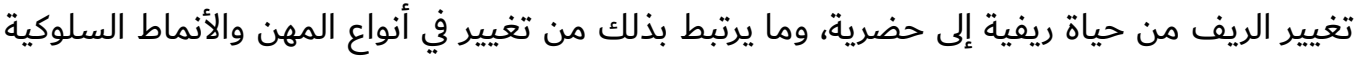
ل اللأفراد. ه- -إحداث تغيرات بيئية من خلال الأخذ بمبدأ التنمية المكانية المستدامة، التي تؤكد على حماية البيئة والتوازن الإقليمي. 
و- -تعميق مستوى الوحدة السياسية والاقتصادية والاجتماعية للبلد، مِمَّا سيعزز من القدرة الدفاعية للبلد تجاه الأخطار الخارجية (1974 (Aiden and Morgan).

\section{ا I-r. مفهوم الاستراتيجية و التخطيط الاستراتيجي المكاني}

يطلق لفظ الإستراتيجية على الأهداف المحددة ووضع البدائل ومقارنة التكاليف والفوائد المرتبطة بها

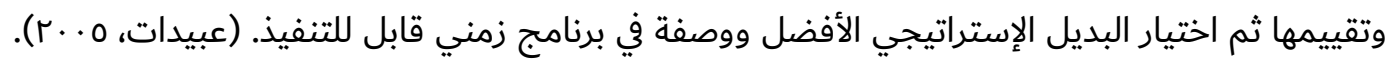

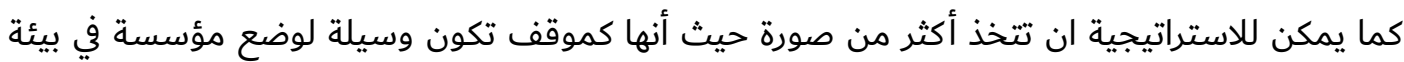

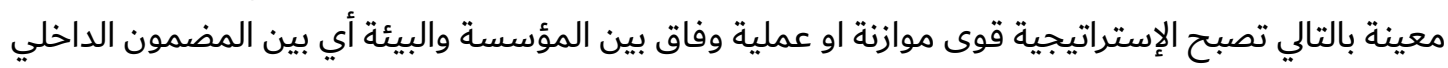

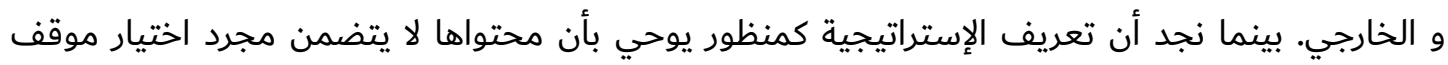

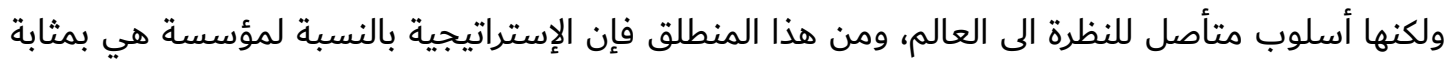

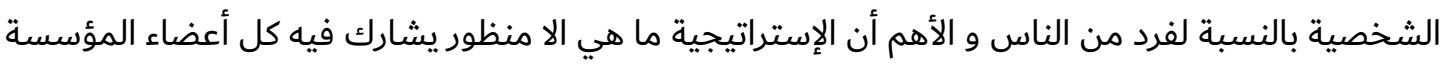

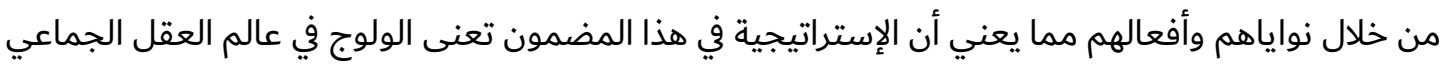

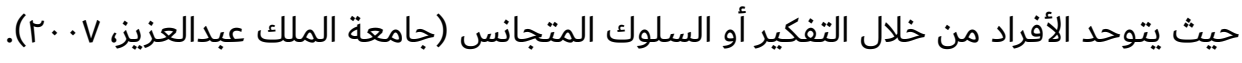

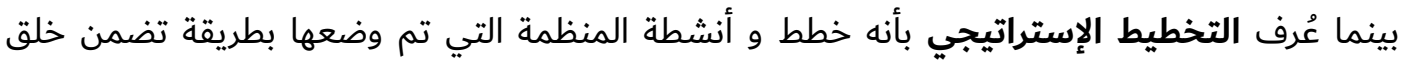

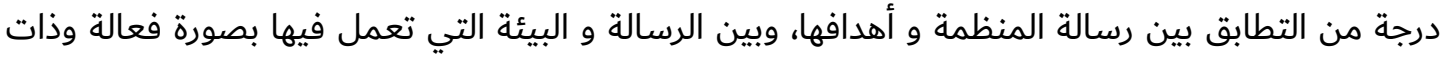

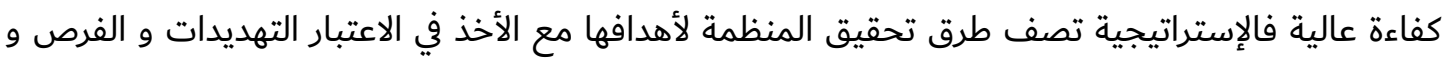

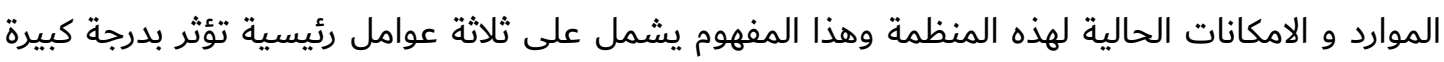

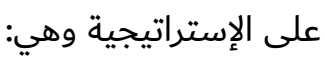

ا. البيئة الخارجية و متغيراتها السياسية و الاقتصادية و الاجتماعية و التكنولوجية. r. الموارد و الامكانات الداخلية خاصة فيما يتعلق بالهيكل التنظيمي والقيادة و القوة و القيم. ب. الأهداف التي تحددها المنظمة وتسعى لإنجازها ضمن إطار زمني محدد. (حبتور، ع · r).

كما ان التخطيط الاستراتيجي يعتمد على سلسلة من العلميات التي تتميز بالديناميكية المرنة ومدى

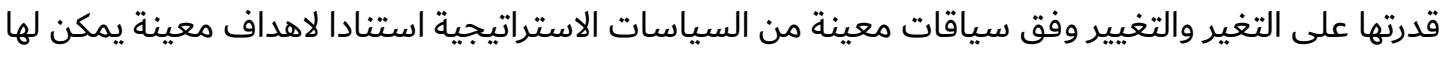

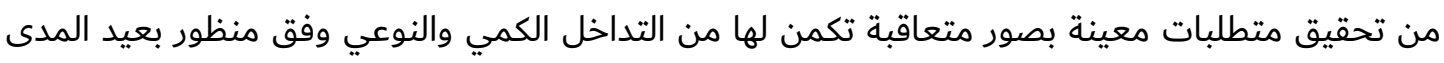

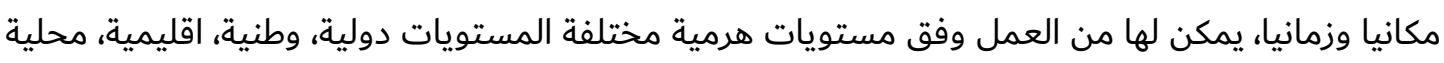

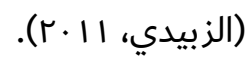

أما التخطيط الاستراتيجي المكاني فهو منظور بعيد المدى يُركز على الحيز المكاني بجميع مستوياته

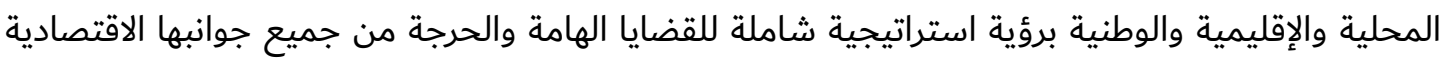

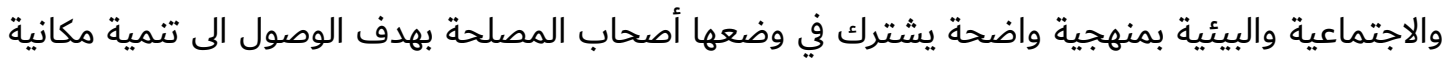
مستدامة (الجارالله و الربح، 17 • r). 


\section{r. ت الدراسات التطبيقية}

نظرا لأهمية دراسة التخطيط الاستراتيجي المكاني فان الدراسات المتعلقة بهذا الموضوع هي محور اهتمام كثير من الباحثين، والهدف من مراجعة الدراسات السابقة هو تسليط الضوء على الجوانب الهامة والمدروسة

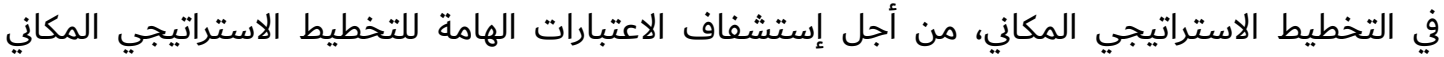

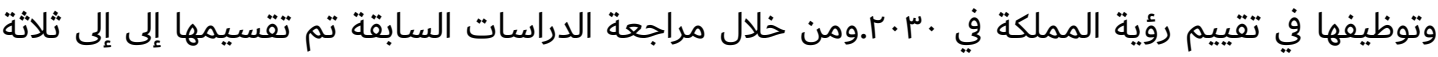
مجموعات:

\section{r-1. المجموعة الاولى: الدراسات ركزت أعتبارات على التنمية البيئية}

هناك عددا من الدراسات العالمية التي ركزت على التخطيط الاستراتيجي المكاني في جانب التنمية البيئية.

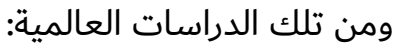

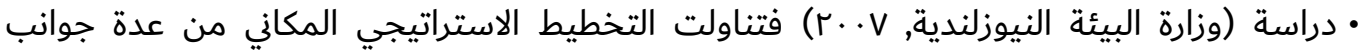
فوضحت انه يهتم بتحديد القضايا الحرجة المكانية وفقا للمتغيرات البيئية و الاجتماعية و الاقتصادية.

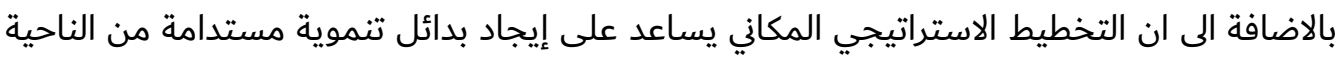

$$
\text { البيئية و الاستراتيجية. }
$$

• اما دراسة (Bussadori, 2011) تناولت التخطيط الاستراتيجي المكاني بالتركيز على الجوانب البيئية

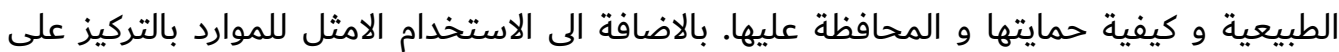

الطاقة المتجددة.

• وتأتي دراسة (منظمة الامم المتحدة, ^• • + التي وضحت أن التخطيط الإستراتيجي المكاني يتميز

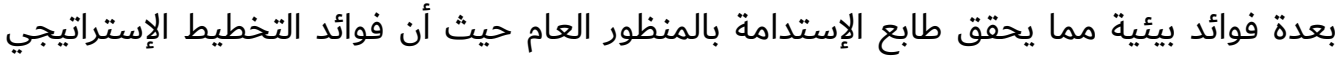

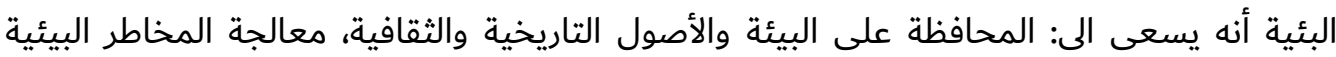
المحتملة، و تشجيع كفاءة إستخدام الطاقة المتجددة غير الضارة في التخطيط وعملية التئه والتمية. • في دراسة (ادريخ، 0 · r) فتناولت اهداف وغايات التخطيط الاستراتيجي المكاني فقد وضحت الدراسة

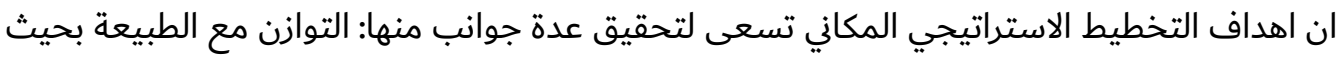

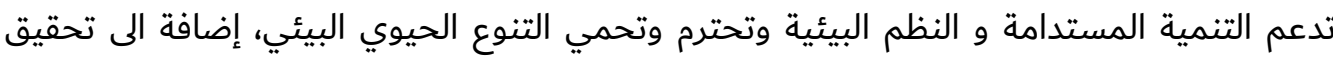
اقتصاد معتمد على المكان فلا يتسبب باستهلاك المصادر الطبيعية.

\section{r-r. المجموعة الثانية: الدراسات ركزت على أعتبارات التنمية الاقتصادية}

• دراسة آدمز وآخرون (Adams \& others 2008) فتناولت سمات التخطيط الإستراتيجي المكاني

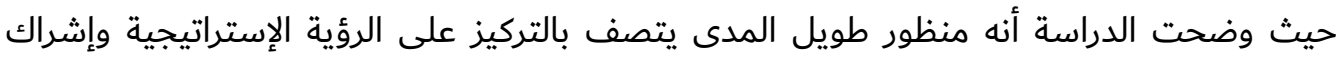

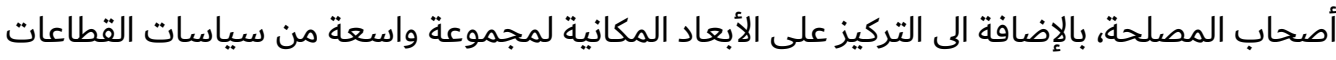
المختلفة من التنمية الاقتصادية و البئية و الاحتماعية.

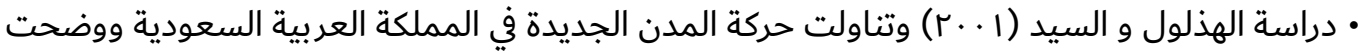
دوافعها المتعددة التي ارتبطت بالسياسة العامة للدولة. كما وضحت الدراسة أن أن طبيعة هذه المداول المدن المدان 
تغيرت وفقا للمتغيرات الاقتصادية التي طرأت على أولويات مراحل التنمية. إلا أن الهدف الضمني

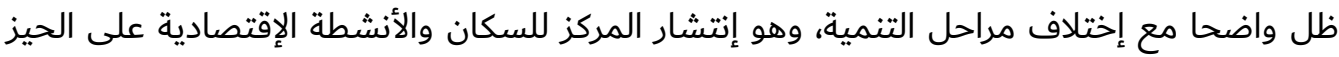

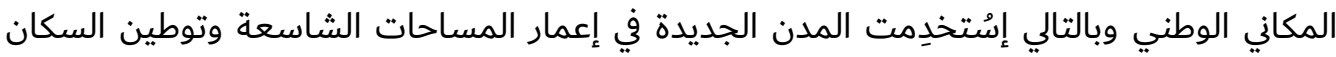

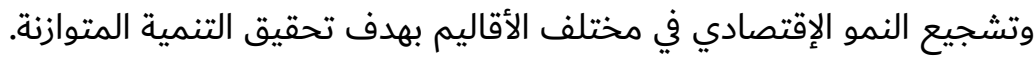

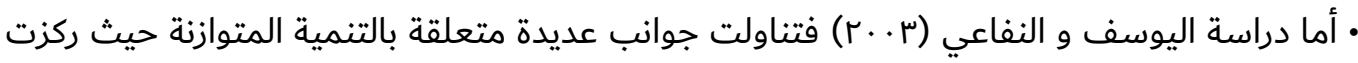

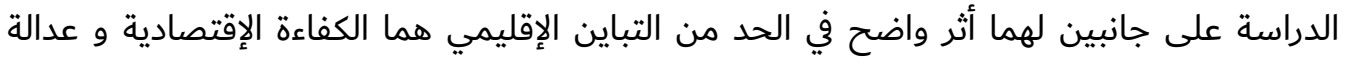

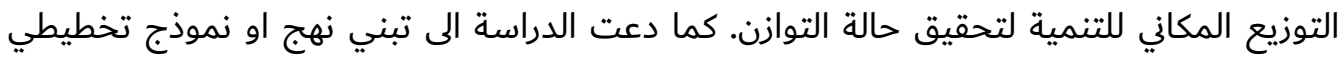
غير تقليدي يساعد على تحقيق معدلات تنموية متقدمة وفقاً للمقومات الإقتصادية و الإجتماعية و المكانية.

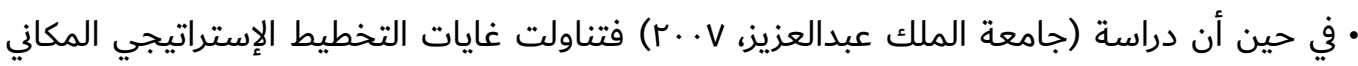

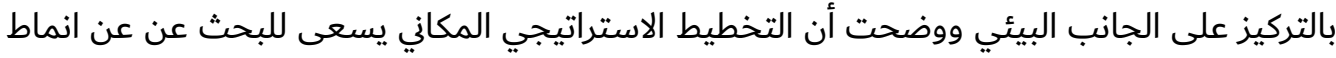

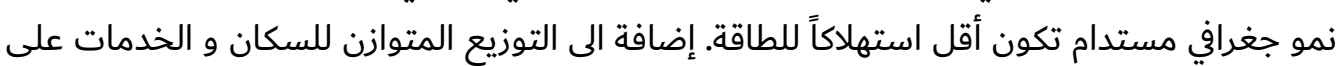
مستوى المناطق والاقاليم و دمج الاعتبارات البيئية و الاقتصادية في عمليات اتخاذ القرار.

\section{ץ-ץ. المجموعة الثالثة: الدراسات ركزت على أعتبارات التنمية الاجتماعية}

• دراسة يونج (Young, 2008) تناولت اهداف التخطيط الاستراتيجي المكاني الذي يسعى لتعزيز

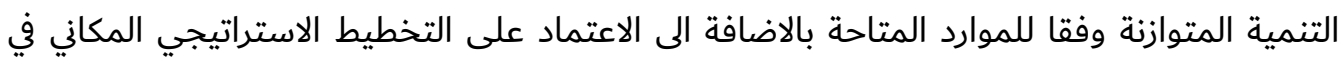

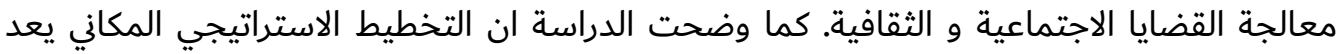

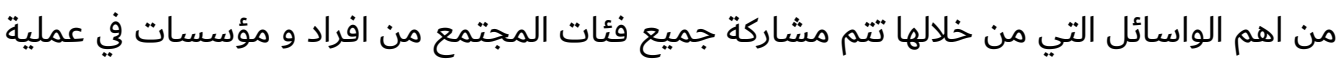
التخطيط و الحفاظ على البيئة.

• أما دراسة البريشتس (Albrechts 2004) فتناولت وظائف التخطيط الاستراتيجي المكاني الذي

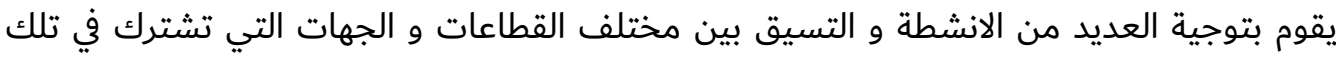

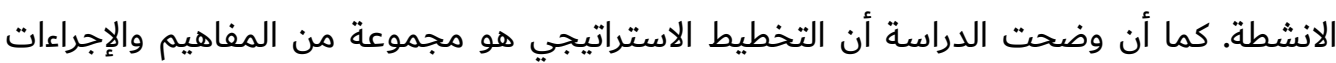
والأدوات المتصلة بالقضايا الاستراتيجية المحددة بجوانبها البيئية و الاجتماعية الاستية و والاقتصادية. • في حين أن دراسة (الجارالله و الربح، 10 · r) تميزت بإهتمامها بالبعد المكاني في الخطط الاستراتيجية

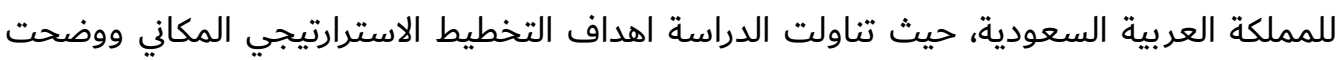

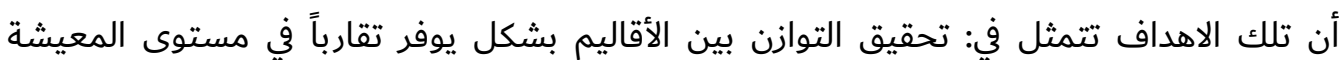
والتقليل من الاتجاهات التلقائية في مجال الهجرة، وتحسين مستوى الإن الأنشطة البيئية و الاجتماعية

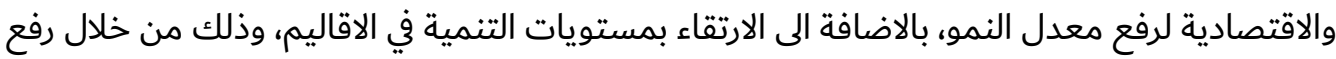

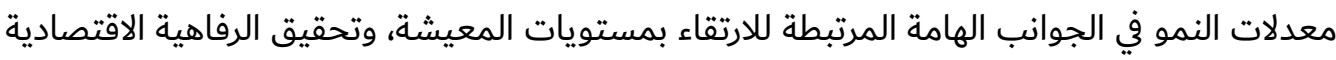

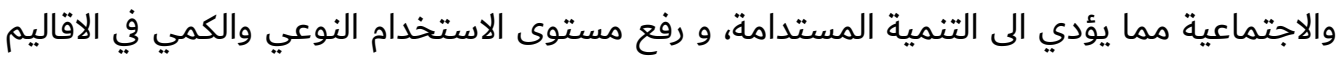

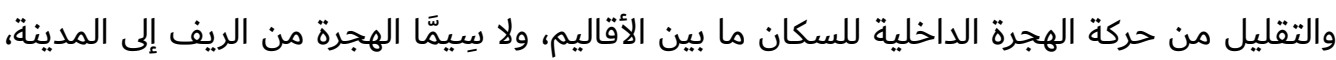
وبذلك تقلل ظاهرة ترييف المدينة من الاتتشار. 


\section{خلاصة مراجعة الدراسات السابقة}

تنوعت الدراسات السابقة من حيث اعتبارات التخطيط الاستراتيجي المكاني، فقد ركزت الدرسات و الاطر

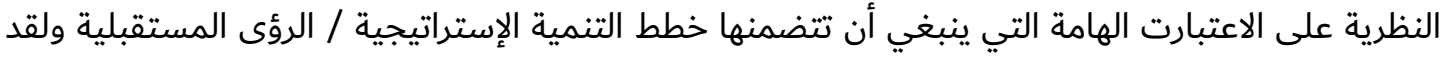
تم استشفاف عشرون اعتبارا هاما للتخطيط الاستراتيجي المكاني كما في الشكل رقم (1) و التي ستكون اساس التحليل في هذه الدراسة.

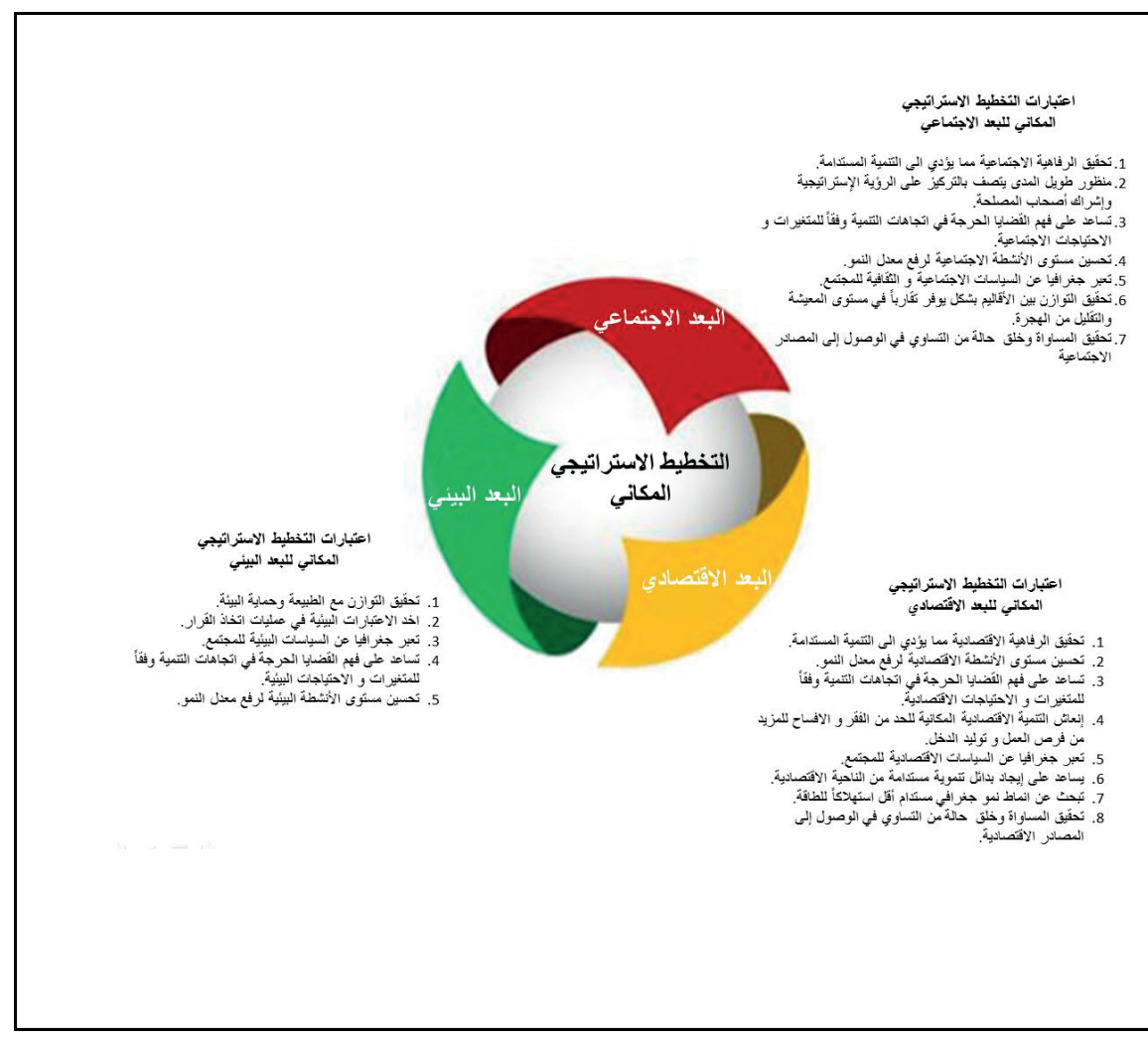

شكل 1: - n

\section{"ا. منهج وأسلوب التحليل}

تتبع الدراسة منهج تحليل المحتوى كمنهج وأسلوب للبحث حيث يعرف تحليل المحتوى بأنه عبارة عن

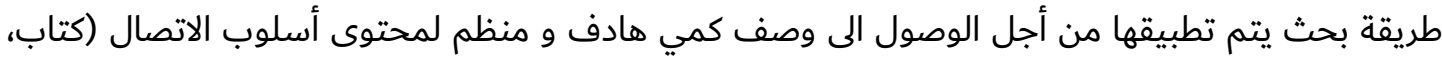

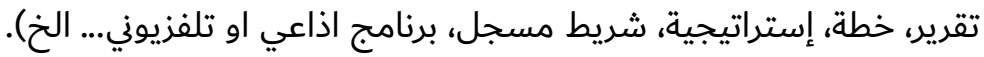

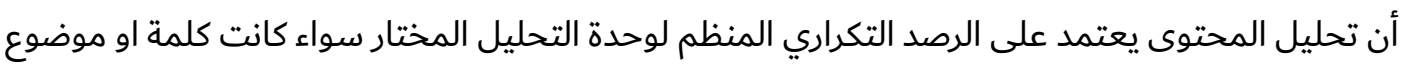
او مفردة او شخصية او وحدة قياس وذلك لتحقيق هدف معين. ويعتمد منهج تحليل المديل المحتوى اساساً

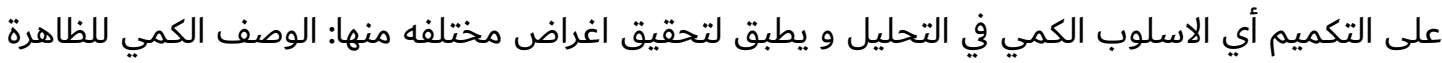
المدروسة من خلال الرصد التكراري لوحدة التحليل المختارة، المقارنة بغرض مقارنة مدى تكرار ظاهرة معينة بظاهرة اخرى، او التقويم لإصدار حكم معين على الاتجاه الغالب حول قضية معينة في مصدر المعلومات.

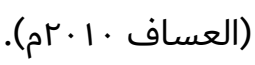


ولتحقيق هدف الدراسة وصف وتحليل وتقويم اهداف التخطيط الاستراتيجي المكاني في رؤية ·ـ ·ـ للممكة

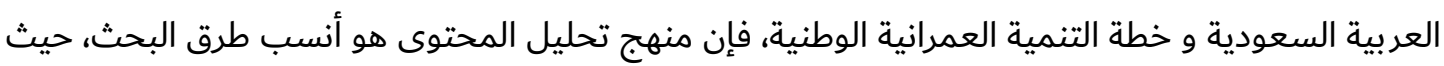
أن منهج تحليل المحتوى سيُطبق بغرض الوصول الى وصف كمي هادف و منظم لمحتوى الحالات الدراسية

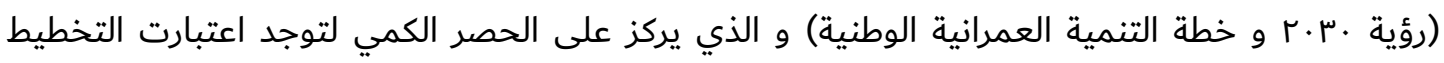

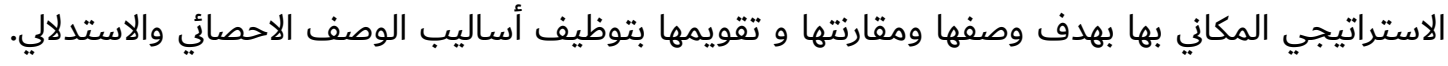

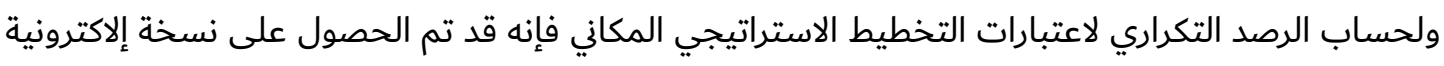

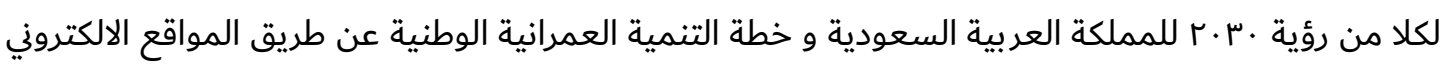

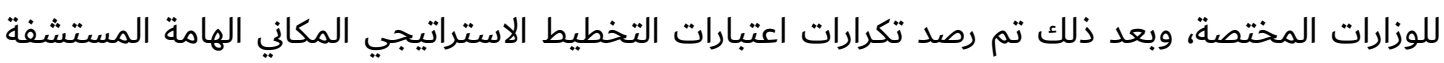

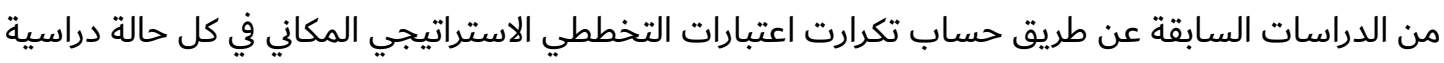
على حده.

\section{ع. وصف وتحليل المعلومات}

بعد تحديد متغيرات الدراسة (اعتبارات التخطيط الاستراتيجي المكاني) تم حساب الرصد التكراري لللاعتبارات في الحالات الدراسية على النحو التالي:

أولا: رصد تكرارات اعتبارات التخطيط الاستراتيجي المكاني في

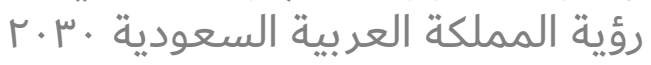

بينات الجدول رقم (1) تبين الحقائق التالية:

ا. أن أجمالي تكرارات الاعتبارات المكانية كانت 1 أن في الرؤية، بينما في الاستراتيجية العمرانية كان .10

تكراراً.

r. أن التكرارات الاقتصادية في الرؤية كانت سه تكرارا تشكل نسبة 90\% من اجمالي التكررات فيها، أما في

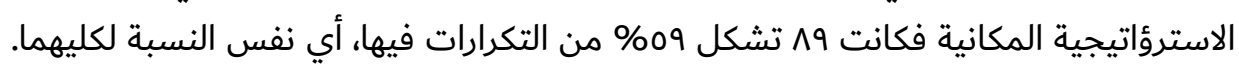

". أن التكرارات البيئية في الرؤية كانت ـ ا تكرارا ت تشكل نسبة | | \% من اجمالي التكررات فيها، بينما في

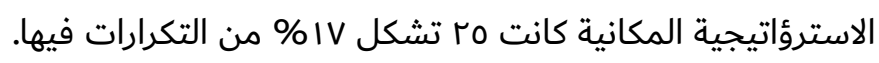

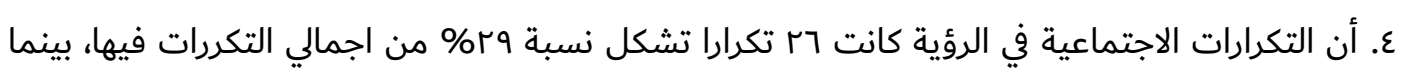

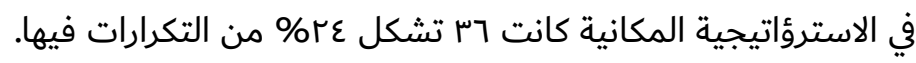

هذه الحقائق توضح أن الاستراتيجية العمرانية تتفوق على الرؤية في أجمالي التكرارات لكل منهما، إضافة

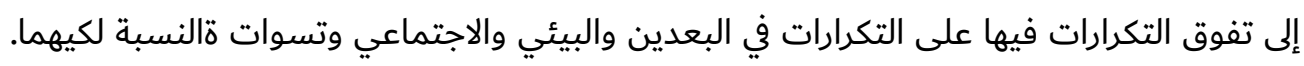

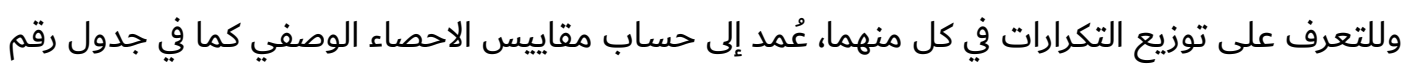




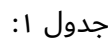

\begin{tabular}{|c|c|c|c|}
\hline & العمر انية & روية 2030 & الالنتراتيجيط \\
\hline 20 & 9 & تحسين مستوى الأنشطة الآتصاية لرفع معدل النهو & \multirow{8}{*}{ أتصاديةرات } \\
\hline 15 & 9 & تجدث عن انماط نمو جغر افي مستشام أقل استهلاكأ للطاقة. & \\
\hline 13 & 8 & إنعاش التنمية الاتقصادية المكانية للحد من الفقر و الافساح للمزيد من فرص العمل و توليد الدخل & \\
\hline 13 & 7 & يساءد على إيجاد بائل تنوية مستامة من الناحية الاقتصادية & \\
\hline 11 & 7 & 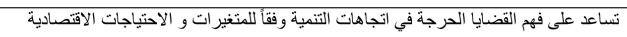 & \\
\hline 9 & 7 & تحقيق المساواة وخلق حالة من النساوي في الوصول إلى المصادر الآتصادية & \\
\hline 5 & 5 & تعبر جغرافيا عن السياسات الاقتصادية للمجتمع & \\
\hline \multirow[t]{2}{*}{3} & 1 & تحقيق الرفاهية الاقتصايةية مما يؤدي الىى التنمية المستشامة & \\
\hline & $\begin{array}{c}89 \\
\% 59\end{array}$ & $\begin{array}{c}53 \\
\% 59\end{array}$ & الالاعتباراتية \\
\hline 11 & 3 & تساعد على فهم القضابا الحرجة في اتجاهات التنّية وفقاً للمتغيرات و الاحتياجات البيئية & \multirow{5}{*}{ بيئة اعتبـارتا } \\
\hline 6 & 2 & اخد الاعتبارات البيئية في عمليات اتخاذ القرار & \\
\hline 5 & 2 & تعبر جغرافيا عن السياسات البيئية للمجتمع & \\
\hline 3 & 2 & تحسين مستوى الأنشطة البيئية لرفع معدل النمو & \\
\hline 0 & 1 & تحقيق التوازن مع الطبيعة وحماية البيئة & \\
\hline & $\begin{array}{c}25 \\
17 \%\end{array}$ & $\begin{array}{c}10 \\
11 \%\end{array}$ & الاعتبارات \\
\hline 14 & 7 & تعبر جغرافيا عن السياسات الاجتماعيةو والثقافية للمجتمع & \multirow{7}{*}{ اجتباعية } \\
\hline 6 & 5 & تساعد على فهم القضايا الحرجة في اتجاهات التنمية وفقاً للمثنيرات و الاحثياجات الاجتماعية & \\
\hline 6 & 5 & تحقيق السساواة وخلق حالة من التُساوي في الوصول إلى الدصادر الاجتماعية & \\
\hline 4 & 4 & منظور طويلي الددى يتصف بالتركيز على الرؤية الإنشر اتيجية وإثر اك أصداب المصلدة. & \\
\hline 4 & 2 & تحسين مستوى الأنشطة الاجتماعية لرفع معدل النهو & \\
\hline 2 & 2 & تحقيق التوازن بين الأقاليع بشكل يوفر تقاربأ في مستوى المبيشة و التقليل من الهجرة & \\
\hline \multirow[t]{3}{*}{0} & 1 & تحقيق الرفاهية الاجتماعية مما يؤدي الىى التنمية المتثدامة & \\
\hline & $\begin{array}{c}36 \\
24 \%\end{array}$ & $\begin{array}{c}26 \\
29 \%\end{array}$ & الالاجتبارات \\
\hline & 150 & 89 & اللألاجنبارات الكماتي \\
\hline
\end{tabular}

جدول r: مقاييس الَّوصف الإحصائي لتكرارات اعتبارات التخطيط الاستراتيجي المكاني في رؤية ·ـ ·r و الاستراتيجية العمرانية.

\begin{tabular}{|r|l|l|}
\hline & \multicolumn{1}{|c|}{$\begin{array}{c}2030 \\
\text { Vision }\end{array}$} & $\begin{array}{c}\text { Urban } \\
\text { Strategy }\end{array}$ \\
\hline $\mathrm{N}$ Valid & 20 & 20 \\
Mean & 4.4500 & 7.5000 \\
Median & $4.2500^{\mathrm{a}}$ & $5.8000^{\mathrm{a}}$ \\
Std. Deviation & 2.79991 & 5.47242 \\
Skewness & .267 & .625 \\
Range & 8.00 & 20.00 \\
Minimum & 1.00 & .00 \\
Maximum & 9.00 & 20.00 \\
Sum & 89.00 & 150.00 \\
\hline
\end{tabular}


بيانات الجدول السابق لقيم مقاييس الاحصاء الوصفي لتكرارات اعتبارات التخطيط الاستراتيجي المكاني في رؤية ·ـ ·r و الاستراتيجية العمرانية تُظهر الحقائق التالية:

ا. أن قيم الوسيط أفضل تمثيلا من المتوسط للاعتبارات المكانية في كل من الرؤية والاستراتيجية

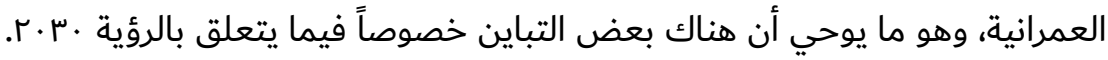

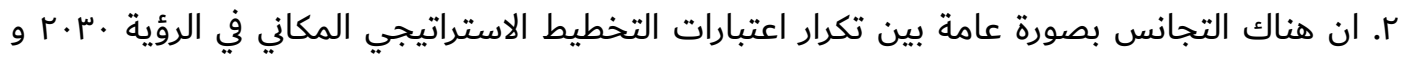
كذلك في خطة التنمية العمرانية الوطنية. وهو ما تُشير إليه القيم المتدينة لمعاملي الالتواء لكل منهما. ب. أن قيمة الوسيط قريبة جداً من قيمة الانحراف المعياري في الاستراتيجية العمرانية، وهو ما يشير إلى تلى

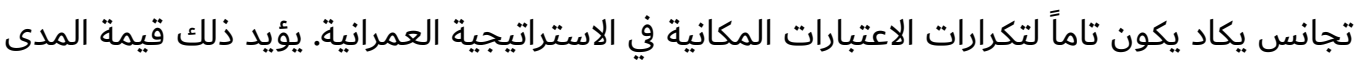

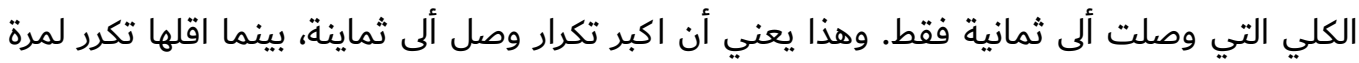
واحدة، أي ان كل الاعتبارات المكانية موجودة في الاساستراتيجية المكانية. ع. أن قيمة الوسيط في الرؤية هي ضعف قيمة الانحراف المعياري، وهو ما يشير إلى ان هناك تباين نسبي

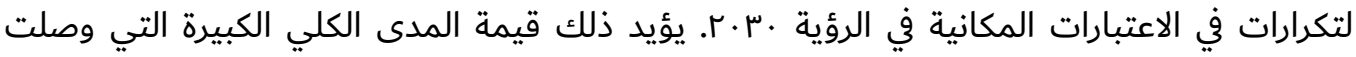

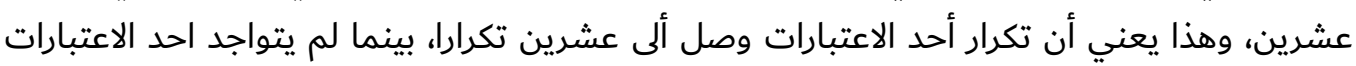
بالمرة في الرؤية.

الحقائق السابقة تبين أن التجانس في تكررا الاعتبارات المكالنية في خطة العمرانية أكثر وضوحاً منه في

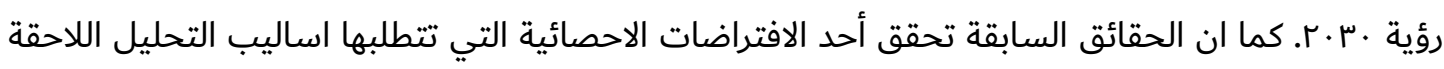

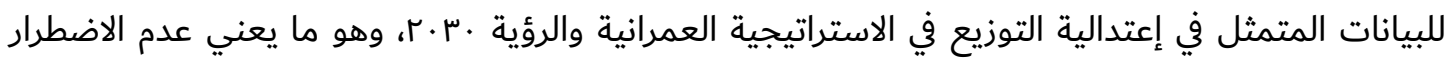

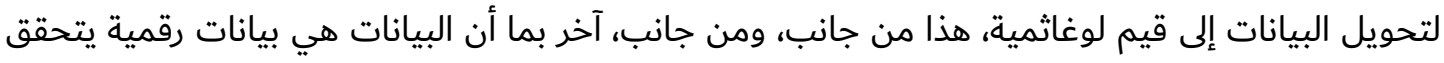
الافتراض المهم الاخر لتحليل.

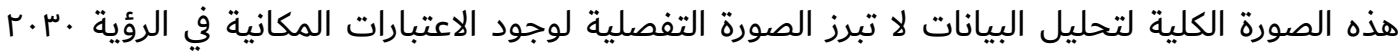

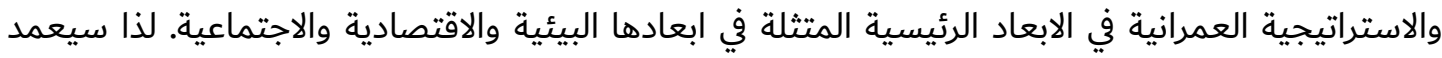

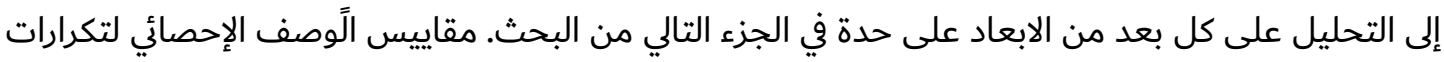

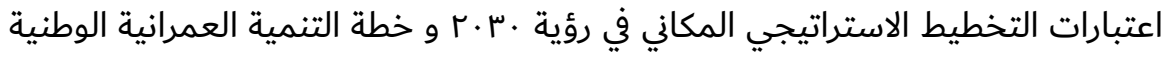

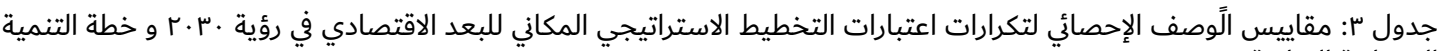
العمرانية الوطنية

\begin{tabular}{|r|r|r|}
\hline Statistics & 2030 Vision & $\begin{array}{r}\text { Urban } \\
\text { Strategy }\end{array}$ \\
& & 8 \\
$\mathrm{~N}$ Valid & 6.6250 & 11.1250 \\
Mean & $7.2500^{\mathrm{a}}$ & $11.6667^{\mathrm{a}}$ \\
Median & 2.61520 & 5.46253 \\
Std. Deviation & $-1.626-$ & $-.006-$ \\
Skewness & 8.00 & 17.00 \\
Range & 53.00 & 89.00 \\
Sum &
\end{tabular}


بيانات الجدول السابق لقيم مقاييس الاحصاء الوصفي لتكرارات اعتبارات التخطيط الاستراتيجي المكاني للبعد الاقتصادي في الرؤية ·ـ ·r والاستراتيجية العمرانية تبين الحقائق التالية:

ا. أن هناك ثمانية أعتبارات تمثل الجانب الاقتصادي تكررت في رؤية ·ـ ·r بمعدل ت V مرات لكل أعتبار.

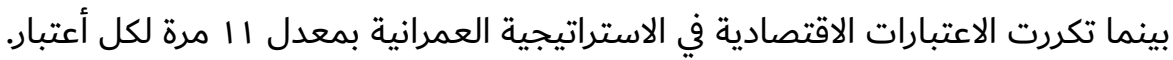

r. ان هناك 9^ تكرارا للاعتبارات الاقتصادية في الاستراتيجية العمرانية، بينما اقتصرت على سأه اعتباراً في

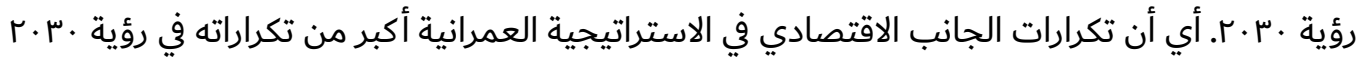
بحوالي الضعف تقريباً.

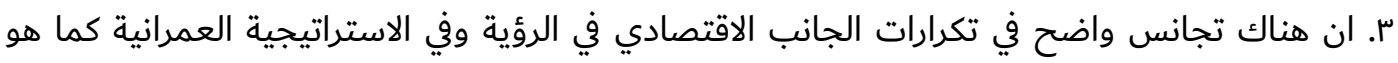

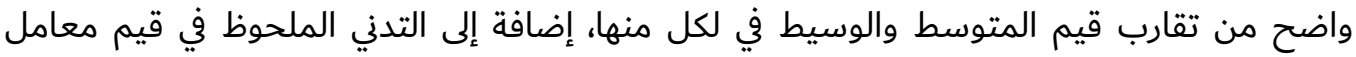

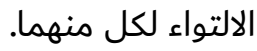

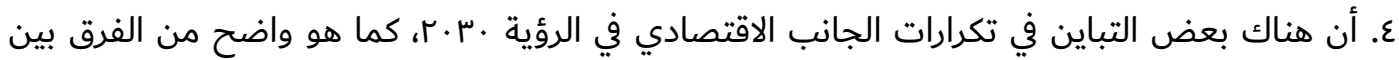

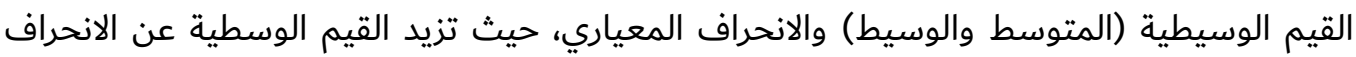
بمقدار الضعف، إضافة إلى أن قمية المدى الكبيرة التي وصلت إلى تسعة للأعتبارات العشرة.

جدول ع: مقاييس الًّصف الإحصائي لتكرارات اعتبارات التخطيط الاستراتيجي المكاني للبعد البيئي في رؤية ·ــr و خطة التنمية

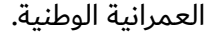

\begin{tabular}{|r|r|r|}
\hline Statistics & 2030 Vision & $\begin{array}{r}\text { Urban } \\
\text { Strategy }\end{array}$ \\
\hline $\mathrm{N}$ & 5 & 5 \\
Valid & 2.0000 & 5.0000 \\
Mean & $2.0000^{\mathrm{a}}$ & $5.0000^{\mathrm{a}}$ \\
Median & .70711 & 4.06202 \\
Std. Deviation & .000 & .522 \\
Skewness & 2.00 & 11.00 \\
Range & 1.00 & .00 \\
Minimum & 3.00 & 11.00 \\
Maximum & 10.00 & 25.00 \\
Sum & &
\end{tabular}

بيانات الجدول السابق لقيم مقاييس الاحصاء الوصفي لتكرارات اعتبارات التخطيط الاستراتيجي المكاني

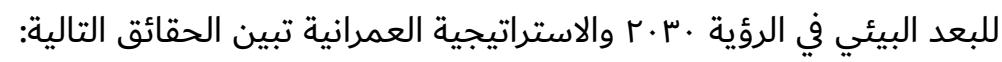

ا. أن هناك خمسة أعتبارات تمثل الجانب البيئي تكررت في الرؤية بمعدل مرتين لكل أعتبار. بينما تكررت

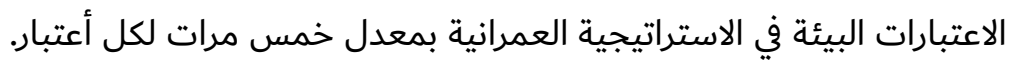

r. أن هناك م r تكرارا للاعتبارات البيئية في الاستراتيجية العمرانية، بينما اقتصرت على · ـ اعتباراً في رؤية

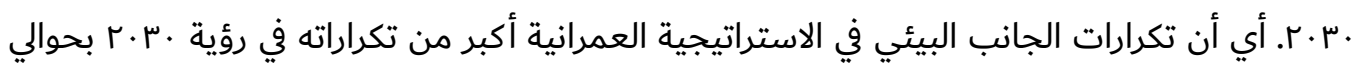




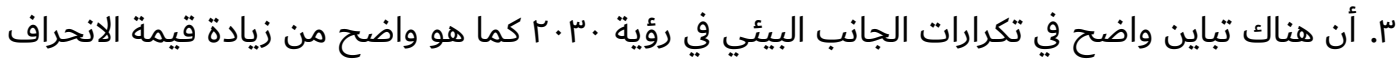
المعياري عن القيم الوسطية، إضافة إلى التدني الملحوظ في قيم معامل الالتواء لكل منهما. ع. أن هناك تجانس تام في تكرارات الجانب البيئي في الاستراتيجية العمرانية كما هو واضح من تساوي

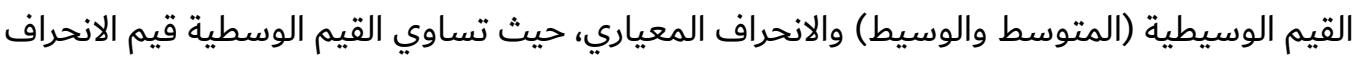
ل اللأعتبارات الخمسة. جدول 0: مقاييس الَّوفف الإحصائي لتكرارات اعتبارات التخطيط الاستراتيجي المكاني للبعد الاجتماعي في رؤية .ب.r. و خطة التنمية العمرانية الوطنية مقايسية

\begin{tabular}{|r|r|r|}
\hline Statistics & 2030 Vision & $\begin{array}{r}\text { Urban } \\
\text { Strategy }\end{array}$ \\
\hline $\mathrm{N}$ & 7 & 7 \\
Valid & 3.7143 & 5.1429 \\
Mean & $4.0000^{\mathrm{a}}$ & $4.5000^{\mathrm{a}}$ \\
Median & 2.13809 & 4.45079 \\
Std. Deviation & .231 & 1.392 \\
Skewness & 6.00 & 14.00 \\
Range & 1.00 & .00 \\
Minimum & 7.00 & 14.00 \\
Maximum & 26.00 & 36.00 \\
Sum & &
\end{tabular}

بيانات الجدول السابق لقيم مقاييس الاحصاء الوصفي لتكرارات اعتبارات التخطيط الاستراتيجي المكاني

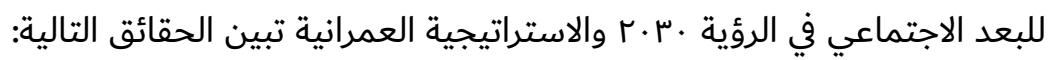

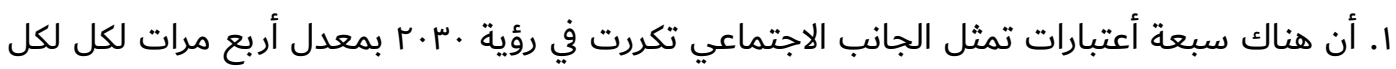

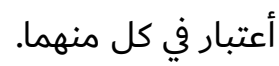

r. أن هناك רץ تكرارا للاعتبارات الاجتماعية في الاستراتيجية العمرانية، بينما اقتصرت على حب اعتباراً

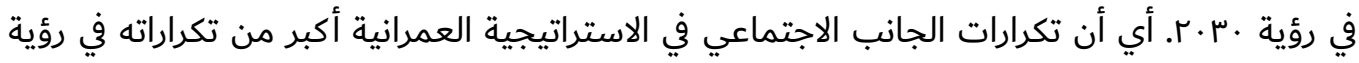

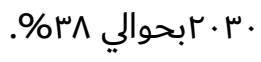

r. أن هناك بعض تباين في تكرارات الجانب الاجتماعي في رؤية .ب.r كما هو واضح من زيادة قيمة الانحراف المعياري عن القيم الوسطية.

ع. أن هناك تجانس تام في تكرارات الجانب الاجتماعي في الاستراتيجية العمرانية كما هو واضح من تقارب

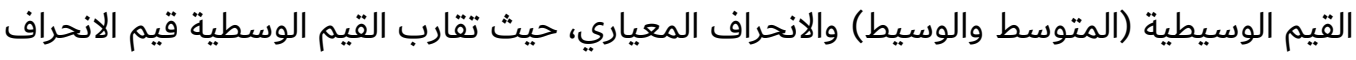
للأعتبارات الخمسة مع وصول قيمة الالتواء غلى الصفر.

لغرض المقارنة بين تكرارات اعتبارات التخطيط الاستراتيجي في رؤية المملكة ·ـ · م مع تكراراتها في خطة

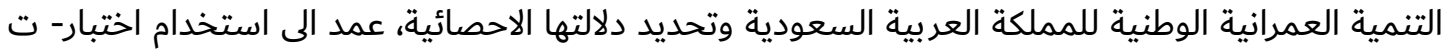

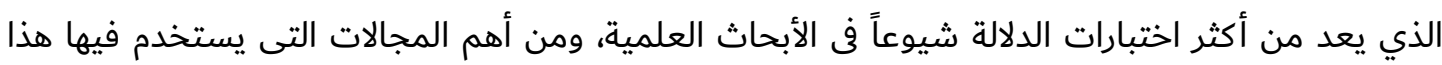

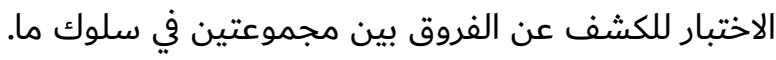




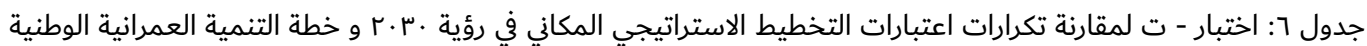

\begin{tabular}{|c|c|c|c|c|c|c|c|c|c|c|}
\hline & \multicolumn{2}{|c|}{$\begin{array}{l}\text { Levene's Test for } \\
\text { Equality of Variances }\end{array}$} & \multicolumn{7}{|c|}{ t-test for Equality of Means } \\
\hline & & \multirow[b]{2}{*}{$F$} & \multirow[b]{2}{*}{ Sig. } & \multirow[b]{2}{*}{$t$} & \multirow[b]{2}{*}{ df } & \multirow[b]{2}{*}{ Sig. (2-tailed) } & \multirow{2}{*}{$\begin{array}{c}\text { Mean } \\
\text { Difference }\end{array}$} & \multirow{2}{*}{$\begin{array}{l}\text { Std. Error } \\
\text { Difference }\end{array}$} & \multicolumn{2}{|c|}{$\begin{array}{l}95 \% \text { Confidence } \\
\text { Interval of the } \\
\text { Difference }\end{array}$} \\
\hline & & & & & & & & & Lower & Upper \\
\hline var001 & $\begin{array}{l}\text { Equal variances } \\
\text { assumed }\end{array}$ & \multirow[t]{2}{*}{9.743} & \multirow[t]{2}{*}{.004} & 2.945 & 36 & .006 & 3.88333 & 1.31856 & 1.20918 & 6.55749 \\
\hline & $\begin{array}{l}\text { Equal variances } \\
\text { not assumed }\end{array}$ & & & 2.861 & 25.745 & .008 & 3.88333 & 1.35755 & 1.09149 & 6.67517 \\
\hline
\end{tabular}

بمراجعة جدول (7) يتبين ان قيمة النسبة الفائية هي (9ع.V) ومستوى دلالتها (ع ...)، هذه القيمة أكبر من مستوى الدلالة (1 ..·)، اي ان القرار الاحصائي يستدعي عدم قبول الفرضية الصفرية وقبول الفرضية البديلة باحتمال 99. ، اي ان هناك اختلاف بين متوسطات تكرارات اعتبارات التخطيط الاستراتيجي المكاني

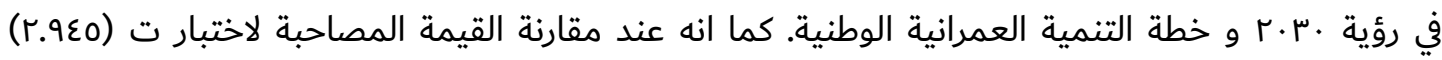

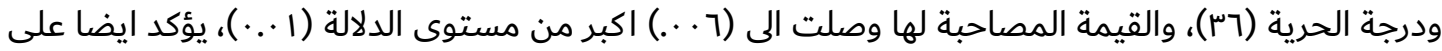

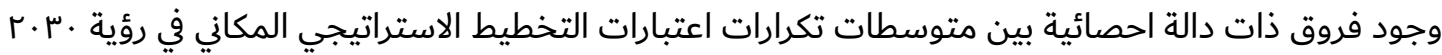

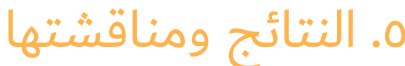

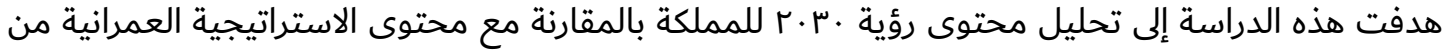

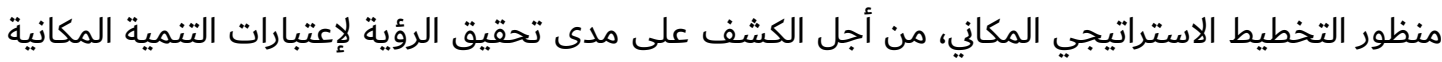

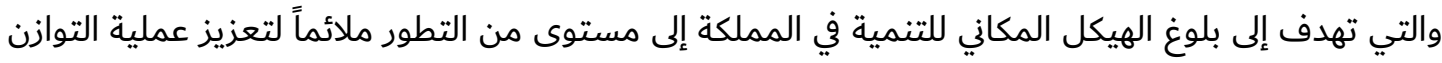
الإقليمي ودعمها بشكل كفء، من خلال دمج الجوانب الأهداف الموقعية بالأهداف القطاعية القومية وبنظرة

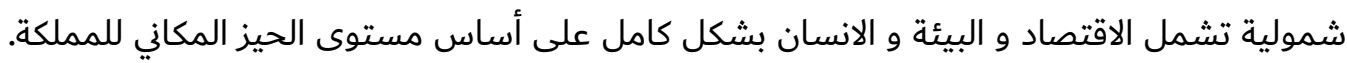
كشف التحليل الكمي لتكررات محتوى الرؤية ·. r.r والاستراتيجية العمرانية، بإن الاستراتيجية العمرانية

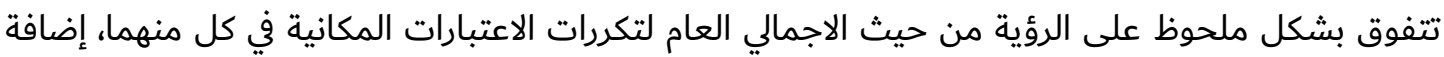

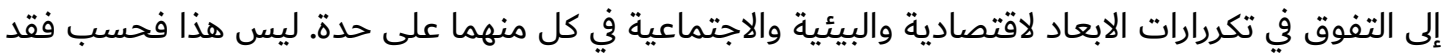

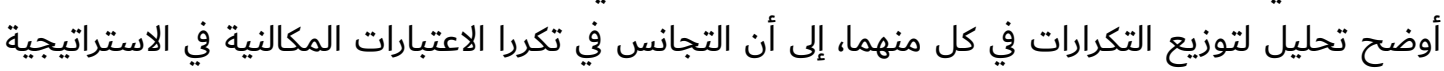

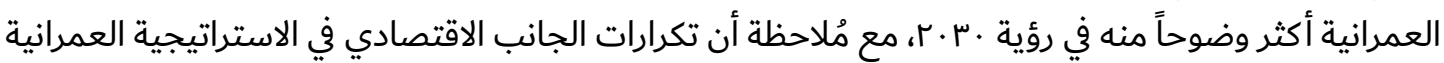

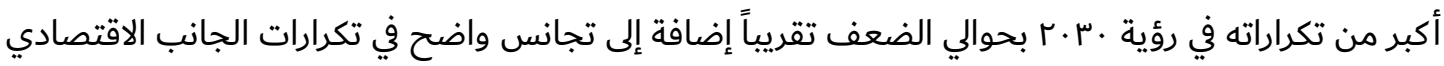
في الرؤية وفي الاستراتيجية العمرانية على حد سواء، مع بعض التباين فئن في تكرارات الجانب الافتصادي في الرؤية $r \cdot \mu \cdot$

اما من حيث البعد البيئي فيلاحظ أن تكرارات الجانب البيئي في الاستراتيجية العمرانية أكبر من تكراراته

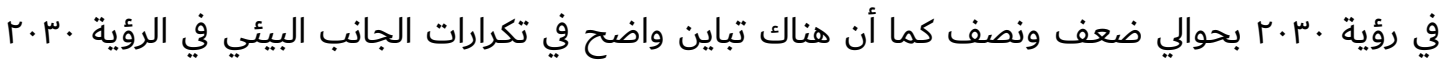

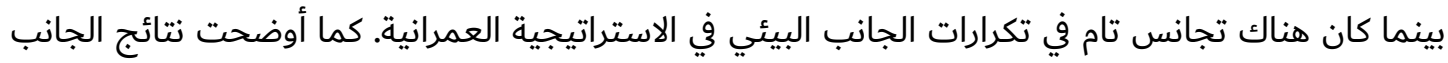

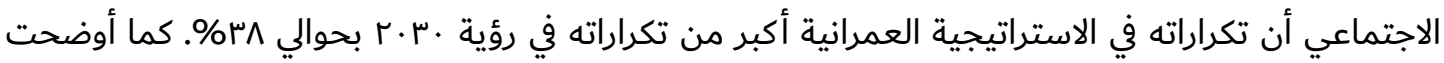

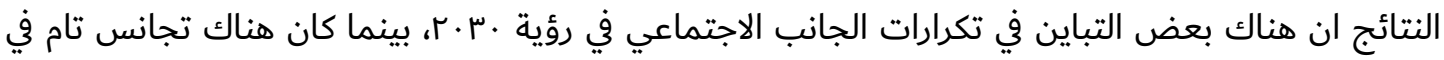
تكرارات الجانب الاجتماعي في الاستراتيجية العمرانية. 
النتائج السابقة تعززها نتائج اختبار"ت" التي تُشير إلى ان هناك فروق بين متوسطات تكرارات اعتبارات

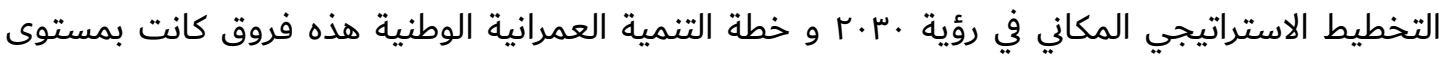

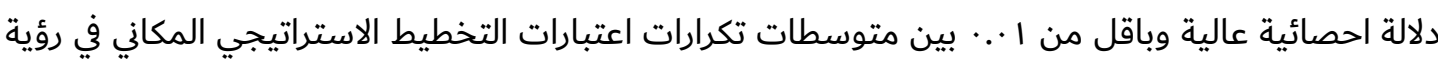
r.r.r.

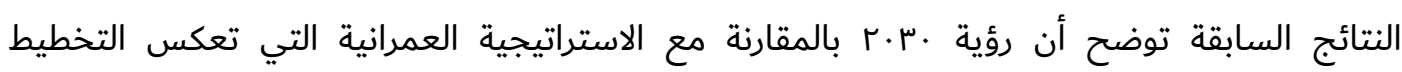

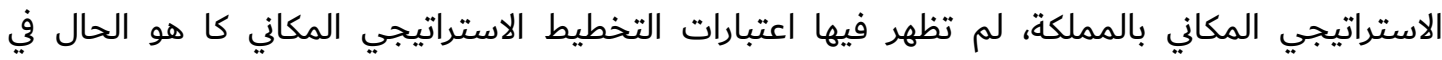

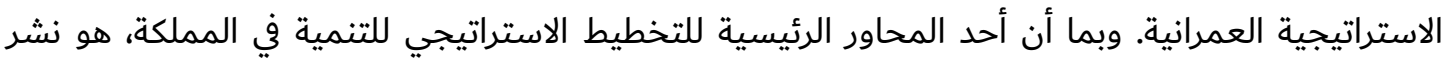

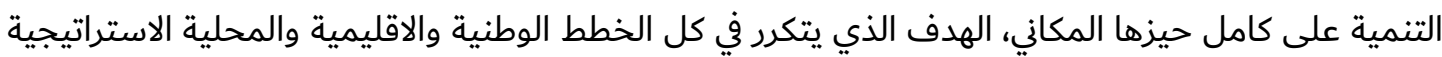

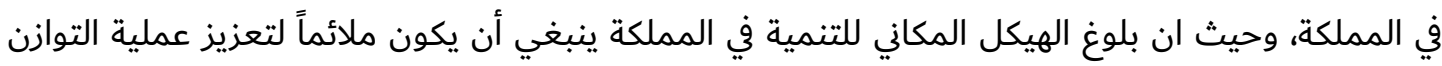

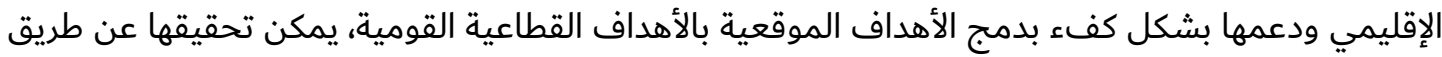

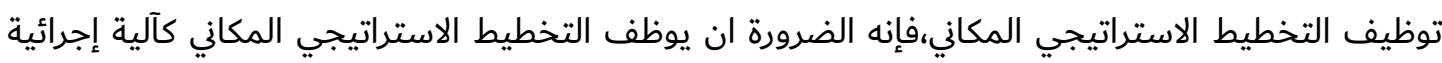

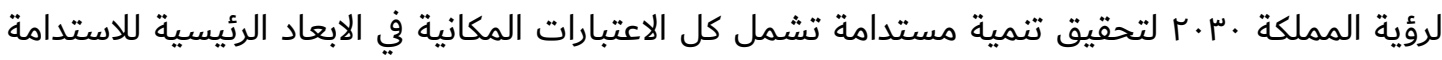
الاقتصادية والاجتماعية والبيئية وتغطي كامل الحيز المكاني للمملكة.

[ [1] ادريخ، مجد عمر، (0 · ·)، استراتيجيات و سياسات التخطيط المستدام و المتكامل لاستخدامات الاراضي

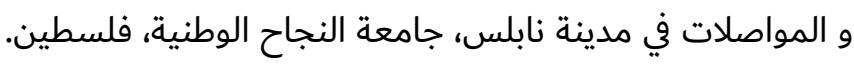

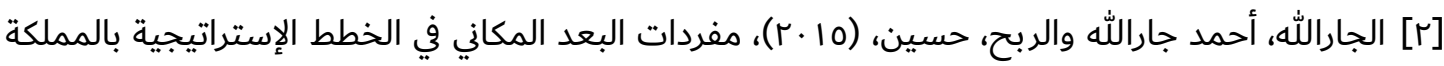

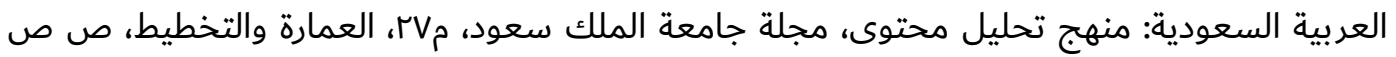

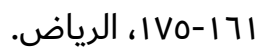

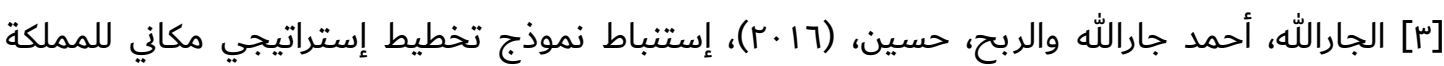

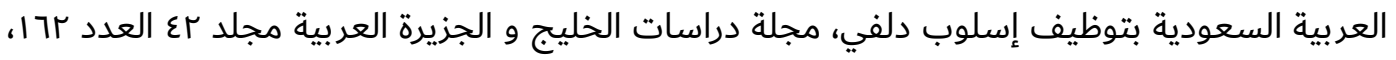
جامعة الكويت، الكويت.

[ع] الزبيدي، مصطفى جليل، (11 · )، التخطيط الاستراتيجي بآليات المدافعة، جامعة بغداد، العراق.

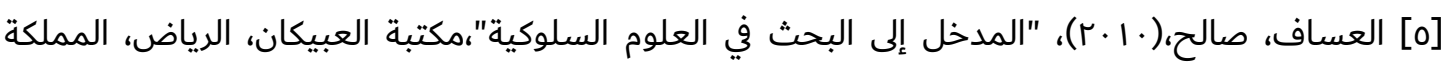
العربية السعودية.

[7] الهذلول، صالح و السيد، عبدالرحمن، (1 .r)، المدن الجديدة بالمملكة العربية السعودية تركيز أم انتشار

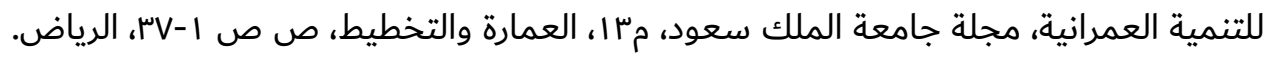

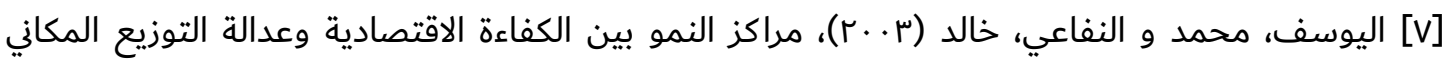

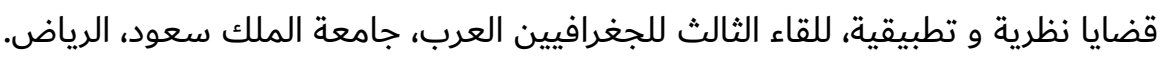

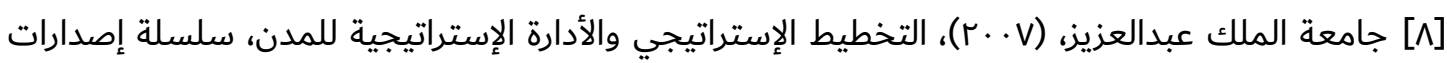
نحو مجتمع المعرفة، الاصدار الخامس عشر. [9] حبتور، عبدالعزيز صالح،(ع · ·)، الادارة الإستراتيجية ادارة جديدة في عالم متغير، دار المسير للنشر و و التوزيع، الاردن.

[· [1] عبيدات، تركي، (0. ·ـ)، التخطيط الإستراتيجي: مفهومه وإطاره الإرشادي ومراحله المختلفة، جامعة العلوم والتكنولوجيا، الاردن. 
[11] Adams, Neil, and others, (2008), Regional Development and Spatial Planning in an Enlarged

[12] Aiden J. and Morgan R.,(1974), Regional Planning A Comprehensive View, Pitman, Great Britain.

[13] Albrechts, Louis, (2004), Strategic (spatial) planning reexamined, Catholic University of Leuven, Belgium.

[14] Bussadori, Virna, (2011), Territorial Cohesion and Spatial Planning Considerations and implications on the local level, Autonomous Province of Bolzano.

[15] Hansen, Niles, (1968) "French Regional Planning", OP. Cit, P. 73

[16] Ministry of Environment,(2007), Building Competitive Cities, New Zealand.

[17] UNITED NATIONS, (2008), Spatial Planning - Key Instrument for Development and Effective Governance with Special Reference to Countries in Transition, New York and Geneva. PP: 2-6.

[18] Young, Greg, (2008), Reshaping Planning with Culture, Macquarie University, Australia

\section{References}

[1] Adriq, Majd Omar, (2005), Strategies and Policies for Sustainable and Integrated Planning for Land and Transport Use in Nablus City, An-Najah National University, Palestine.

[2] Al-Jarallah, Ahmad Jarallah and Al-profit, Hussein, (2015), Spatial Dimension in Strategic Plans in Saudi Arabia: Content Analysis Approach, King Saud University Journal, 27, Architecture and Planning, pp. 161-175, Riyadh.

[3] Al-Jarallah, Ahmad Jarallah and Al-Farah, Hussein, (2016), Development of a spatial strategic planning model for the Kingdom of Saudi Arabia using the Delphi method, Journal of Gulf Studies and Arabian Peninsula Volume 42, No. 162, Kuwait University, Kuwait.

[4] Al-Zubaidi, Mustafa Jalil, (2011), Strategic Planning of Defensive Mechanisms, University of Baghdad, Iraq.

[5] Assaf, Saleh, (2010), "Introduction to Research in Behavioral Sciences", Obeikan Library, Riyadh, Saudi Arabia.

[6] Adams, Neil, and others, (2008), Regional Development and Spatial Planning in an Enlarged

[7] Aiden J. and Morgan R.,(1974), Regional Planning A Comprehensive View, Pitman, Great Britain. 
[8] Albrechts, Louis, (2004), Strategic (spatial) planning reexamined, Catholic University of Leuven, Belgium.

[9] Bussadori, Virna, (2011), Territorial Cohesion and Spatial Planning Considerations and implications on the local level, Autonomous Province of Bolzano.

[10] Hansen, Niles , (1968) "French Regional Planning", OP. Cit, P. 73

[11] Ministry of Environment,(2007), Building Competitive Cities, New Zealand.

[12] UNITED NATIONS, (2008), Spatial Planning - Key Instrument for Development and Effective Governance with Special Reference to Countries in Transition, New York and Geneva. PP: 2-6.

[13] Young, Greg, (2008), Reshaping Planning with Culture, Macquarie University, Australia 